\title{
MPC5602P-Based Hardware Circuit Design for Electric Power Steering System
}

\author{
Qinghe Liu ${ }^{\mathrm{a}}$, Qingyang $\mathrm{Xu}{ }^{\mathrm{b}}$, Weiguang Kong ${ }^{\mathrm{c}}$ and Shouzhi Liu ${ }^{\mathrm{d}}$ \\ School of Automobile Engineering, Harbin Institute of Technology at Weihai, Weihai 264209, China

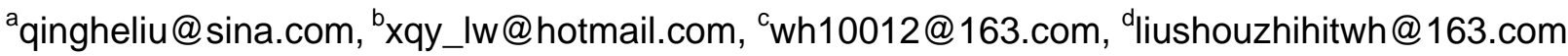

Keywords: EPS, MPC5602P, Modular Design, Induction Motor Vector Control.

\begin{abstract}
This paper analyzes the design requirements of high-performance EPS controller, on the basis of which, we select Freescale Power Architecture ECU MPC5602P special for EPS. By the means of Modular Design, we propose a new design for EPS controller hardware, which focuses on MPC5602P minimum system, power supply module, three-phase induction motor inverter module and sensor signal conditioning module. Meanwhile, we completed EPS controller program design process in the light of the induction motor vector control theory. Finally, our controller realized the control functions of the electric power steering motor after it was debugged.
\end{abstract}

\section{Introduction}

Following the three themes of automotive development-safety, energy saving, environmental protection, Electric power steering system (EPS), with its energy conservation and emissions reduction, convenient installation, low maintenance cost and good assist characteristic, gradually occupies the steering market and becomes a hot research in automotive electronics industry in recent years. With the emergence of Lane Keeping, Automatic Parking, Pull-Drift Compensation and other driving assistant system, EPS cannot work together with them unless its quick response, low power consumption and excellent stability. In addition, the steering system trends to the Steer-by-Wiresimilar to EPS. And INDUSTRIE 4.0 put forward "software is the future of industry", which means EPS needs powerful hardware support to run the huge program smoothly, and thus the EPS research focused on the controller hardware design is particularly important.

Freescale and STMicroelectronics have jointly developed Power Architecture ${ }^{\mathrm{TM}}$ microcontroller, which can satisfy the need to the auto equipping with 32 bit ECU. MPC560xP series ECU are specially developed for the chassis and safety, while MPC5602P is for EPS and airbag. In this paper, the EPS system controller hardware is designed by using MPC5602P as ECU, combining with Freescale power management chip MC33905S and 3-phase MOSFET pre-driver chip MC33937.

\section{EPS controller hardware architecture}

The EPS controller should implement the following functions:

(1) Generating and monitoring the supply voltage component;

(2) Monitoring and conditioning steering torque signal and the steering angle signal;

(3) Receiving vehicle speed and engine speed signal via CAN or FlexRay ${ }^{\mathrm{TM}}$ protocol;

(4) Calculating the required assist torque;

(5) Motor speed signal conditioning and vector control;

(6) Producing the PWM signal to regulate and control the 3-phase motor driver MOSFETs.

To meet the basic functional requirements of EPS, its controller is divided into four main sections:

(1) MPC5602P minimum system. Minimum system is facilitate the early design debugging.

(2) Power supply module. Convert $12 \mathrm{~V}$ to stabilize 5V and monitor the voltage state. Promptly cut off power supply when a malfunction occurs, ensure the controller is not damaged.

(3) Motor control inverter module. Driving MOSFETs to generate PWM modulation wave, at the same time, the motor over-current condition is detected and an error signal is fed directly to the ECU. 
(4) Sensor signal conditioning. Acquisition signals of EPS controller are divided into digital signal and analog signal. Digital signal can be directly used for ECU after conditioning. Remaining signals, analog signals, are collected by the ADC after conditioning.

According to the functional requirements, the hardware architecture is designed in Figure 1.

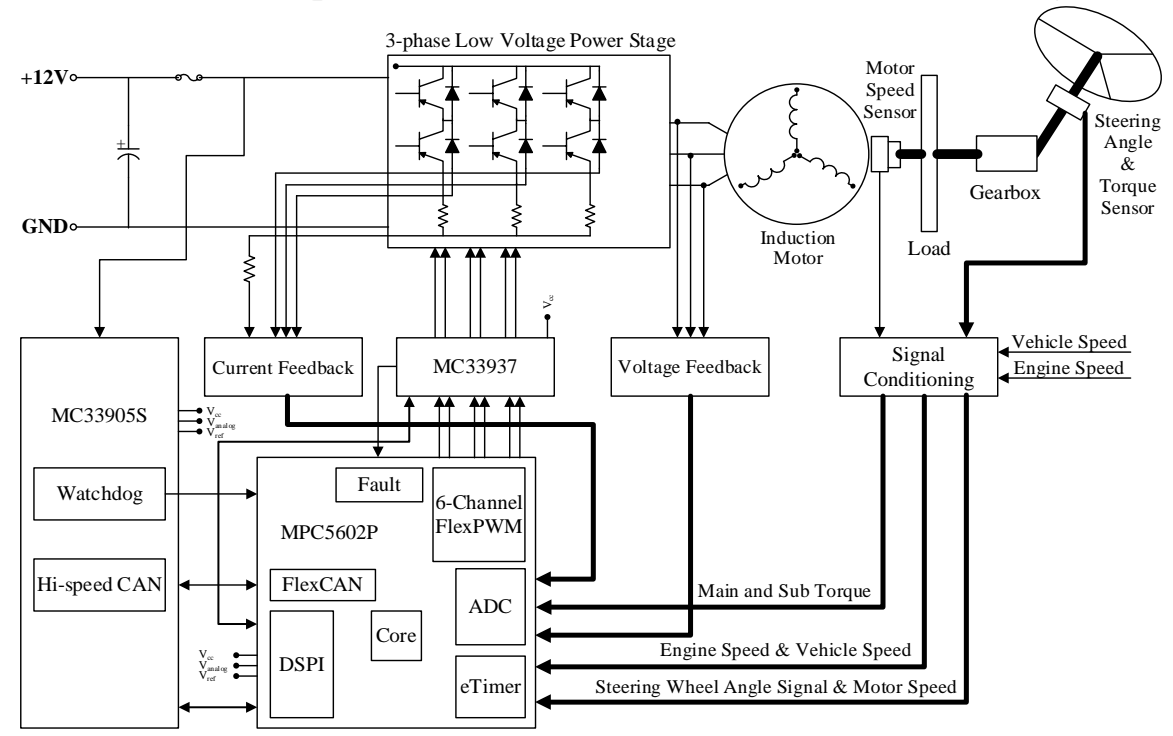

Fig. 1 EPS controller hardware architecture

\section{EPS controller modular design}

\subsection{MPC5602P minimum system design}

MPC5602P is a 32-bit power Architecture ECU, which has a 9-channel Flex PWM, 5-channel Enhanced Timer, 10-channel 16-bit ADC, 20K RAM and 256K Flash program memory. And the 64 LQFP packages can meet the system requirements. Table 1 is the resource allocation of MPC5602P.

Table 1 ECU resource allocation

\begin{tabular}{|c|c|c|c|c|c|}
\hline \multicolumn{2}{|r|}{ ADC } & \multicolumn{2}{|c|}{ Flex PWM } & \multicolumn{2}{|c|}{ EIRQ } \\
\hline $\mathrm{AD} 0,2,4$ & Voltage feedback & $\mathrm{A} 0 \sim 2, \mathrm{~B} 0 \sim 2$ & PWM drive & EIRQ3 & Over-current \\
\hline AD1, 3 & torque & \multicolumn{2}{|c|}{ GPO } & EIRQ5 & Error interrupt \\
\hline $\mathrm{AD} 12 \sim 14$ & Current feedback & GPIO9, 60 & Enable 33937 & EIRQ17 & Interrupt request \\
\hline AD6 & Motor speed & GPIO55 & Reset 33937 & EIRQ13, 14, 18 & Low-voltage \\
\hline \multicolumn{2}{|r|}{ Timer } & \multicolumn{2}{|c|}{ GPI } & \multicolumn{2}{|c|}{ DSPI } \\
\hline ETC5 & Steering angle & GPIO5, 15, 16 & digital signal & CS1, 2 & Chip select \\
\hline
\end{tabular}

The basic circuit of MPC5602P minimum system is designed according to Freescale product TRK-USB-MPC5602P. It should be noted that there are 3 parts to power MPC5602P: $\mathrm{V}_{\text {сc }}$ (8-groups $5 \mathrm{~V} / 3.3 \mathrm{~V}$ power pins and 6-groups $1.2 \mathrm{~V}$ core power pins); $\mathrm{V}_{\text {analog }}$ (a group of ADC supply pins); $\mathrm{V}_{\text {ref }}$ (voltage regulator power and a combination of external NPN transistor to generate $1.2 \mathrm{~V}$ voltage). Meanwhile, every part of the decoupling capacitors should be close to the pin.

Using the software RAppID Init for MPC560xP, we can define the function of each pin, set the parameters of each module and generate initialization codes. And this codes can be imported into CodeWarrior project via the software rsp2cw10.

\subsection{Power supply module design}

Power module is very important for EPS controller stability. The most important function is able to supply the controller stable voltage under complex environmental interference. In this paper we selects Freescale System Basis Chip MC33905S. The power supply module is shown in Figure 3.

It should be noted that the DBG pin connection method affect the circuit operation mode:

(1) DBG connected directly to ground, the chip disables to use Debug mode. In order to ensure the normal operation of MC33905S, a periodic watchdog refresh must occur via SPI. 
(2) DBG detects a voltage between 8.0 and 10.0V, the chip activates the Debug mode. The chip can also work as usual without the watchdog refresh in this case, which facilitates the debugging.

In this design, we set the chip to the Debug mode. Specially, 8.0 to $10.0 \mathrm{~V}$ voltage divider circuit differs more with the calculated values. Therefore, in the case of $12.0 \pm 1.0 \mathrm{~V}$ power supply, we choose $47.0 \mathrm{~K}$ and $2.7 \mathrm{~K}$ voltage divider circuit after being tested, which can ensure the DBG pin get $8.6 \mathrm{~V}$ to $9.8 \mathrm{~V}$ and the chip work in the state of Debug.

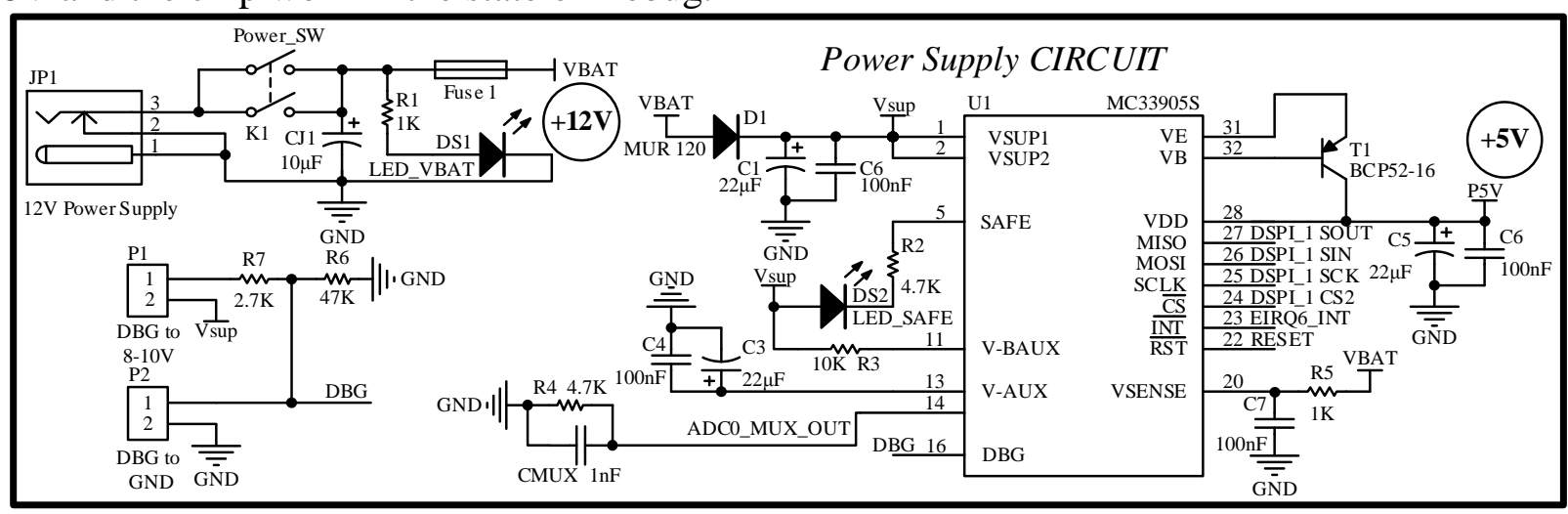

Fig. 2 power supply module

\subsection{Motor control inverter module}

Motor inverter control module is the core of the EPS controller. It has MOSFETs pre-drivers and inverter two parts. MOSFETs pre-driver is implemented by Freescale MC33937. This chip operating voltage between a range of 6.0 to $58 \mathrm{~V}$, covering $12 \mathrm{~V}$ and $42 \mathrm{~V}$ used in the vehicle. Meanwhile, the chip, having more than 1A gate drive capability, can protect external FETs by reverse charging and can be programmed via SPI commands to feed back the state of the external FETs. To improve the system integration, inverter circuit selects Infineon Dual N-channel IPG20N06S4-15 as the high and low side of each phase. IPG20N06S4-15 has a maximum 60V drain-source voltage and a maximum 20A drain-source current. Maximum using temperature can reach 175 degrees. The motor control inverter module is shown in Figure 3.

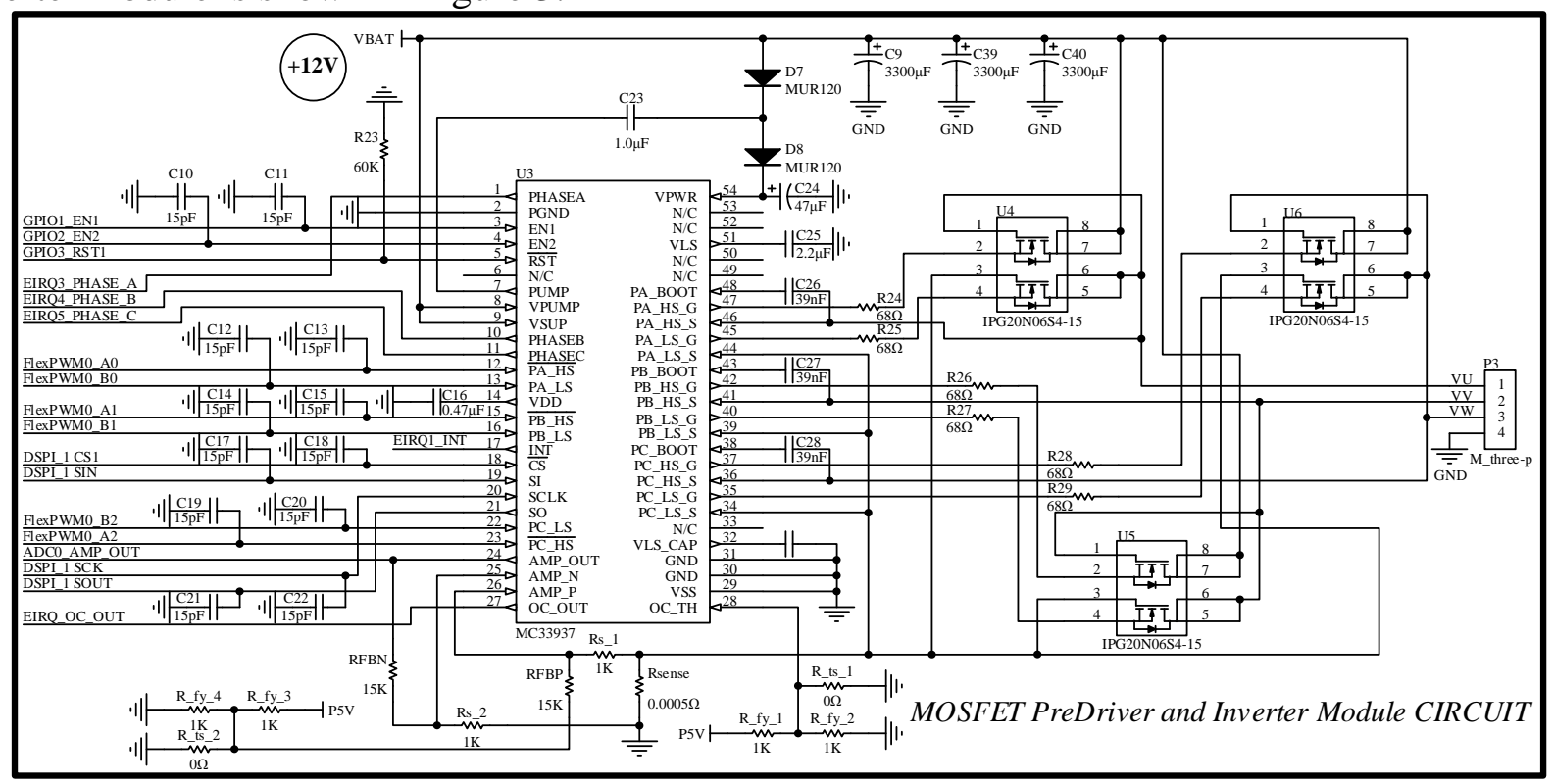

Fig. 3 motor control inverter module

In the circuit, the capacitors C26, C27, C28 directly connected by PX_BOOT pin is the 3-phase boot capacitor $\mathrm{C}_{\mathrm{X} \_ \text {воот. }}$. Boot capacitor value is calculated as follows:

$$
\begin{aligned}
& C_{X_{-} \text {Boot }}=(10 \sim 20) \cdot C_{G S} \\
& C_{i S S}=C_{G S}+C_{G D} \\
& C_{o S S}=C_{G D}+C_{D S} \\
& C_{r S S}=C_{G D}
\end{aligned}
$$


$\mathrm{C}_{\mathrm{iSs}}$ is the input capacitance value of the drain-source open circuit; $\mathrm{C}_{\mathrm{oss}}$ is common source output capacitance value; $\mathrm{C}_{\mathrm{rSS}}$ is reverse transfer capacitance value, these three data provided by MOSFET manufacturers. Thereby calculating the interelectrode capacitance value $\mathrm{C}_{\mathrm{GS}}, \mathrm{C}_{\mathrm{DS}}, \mathrm{C}_{\mathrm{DS}}$.

Finally, we get $\mathrm{C}_{\mathrm{X} \_ \text {воот }}=19.6 \sim 39.2 \mathrm{nF}$. According to the requirements of the low-side MOSFET rise time:

$$
5 \cdot C_{\text {Boot }}\left(R_{\text {SENSE }}+R_{\text {DSON_LS }}\right) \ll 100 n s
$$

Thus, the low-side MOSFET responses fast if boot capacitor value is high. Taking into account the 0805 Surface Mounted Devices package capacitance values within this range, ultimately we use 39nF as the boot capacitor value.

\subsection{Signal conditioning module}

Sensor signal conditioning module is divided into digital signal conditioning and analog signal conditioning, the following are introduced successively.

EPS controller conditioning digital signals including: vehicle speed signal, engine speed signal and the steering angle signal. Signal is sent to the ECU timer or general-purpose input after being filtered by Opt coupler and shaped by Schmitt trigger. Capacitor is used to anti-interference. Regulator tube is used to limiting protection.

EPS controller conditioning analog signals including: motor phase voltage feedback and current feedback, which can present the state of motor running so that complete the motor vector control. The torque sensor output signals is also the $0 \sim 5 \mathrm{~V}$ analog signals. It can be processed in the same circuit.

The signal conditioning module is shown in Figure 4.

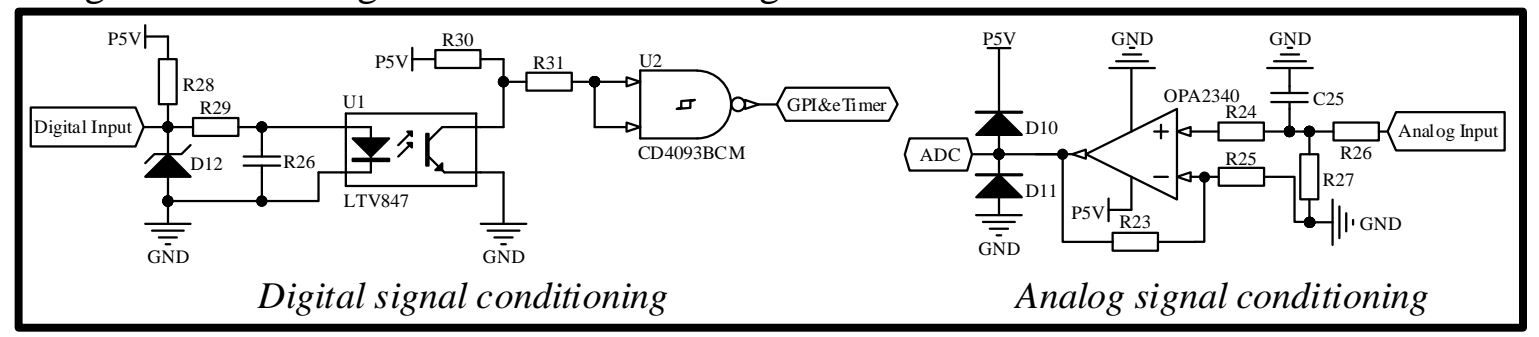

Fig. 4 signal conditioning module

\section{EPS controller programming}

According to the theory of induction motor vector control model shown in Figure 5, The program design of EPS controller can be divided into ECU initialization, the sensor signal acquisition, motor state acquisition (voltage feedback, current feedback and motor speed), fault diagnosis and assistant mode selection (power assisted control, returnable control and damping control).

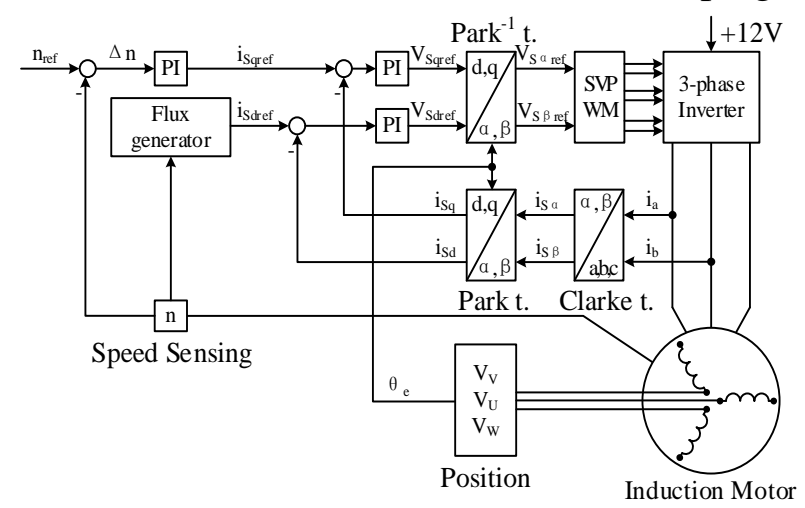

Fig. 5 Induction motor vector control block diagram

ECU initialization including reuse pins define, initialize Flex PWM, ADC and DSPI, set the timer and interrupt priority. After ECU is initialized and powered, ECU will diagnose EPS controller status according to the torque sensor signal and steering angle sensor signal. If the EPS works well, ECU will determine the state of the steering operation via the torque and the steering angle sensor data, and then, ECU selects the assistant mode. Meanwhile, EPS determines motor target rotational speed by 
the assist characteristics curve which is related to the vehicle speed and engine speed. PI control the deviation of the motor speed and the target speed to get the target current. At the same time, using the 3-phase voltage feedback determines the rotor position. PI control the deviation between the feedback current and the target current value to get the inverter output voltage. Then, enables MOSFETs pre-driver output PWM signal to controls the operation of induction motor.

When MC33937 detects the overcurrent, phase voltage less than $50 \%$ or other adverse condition, MC33937 sends an interrupt request to the ECU, at this time, ECU will produce the reset operation signal to MC33937. If MC33937 continuously ask for an interrupt request, disable the MOSFETs pre-driver output. After the ECU reset or repowered, ECU waiting for the torque angle signal. This program flow chart is shown in Figure 6.

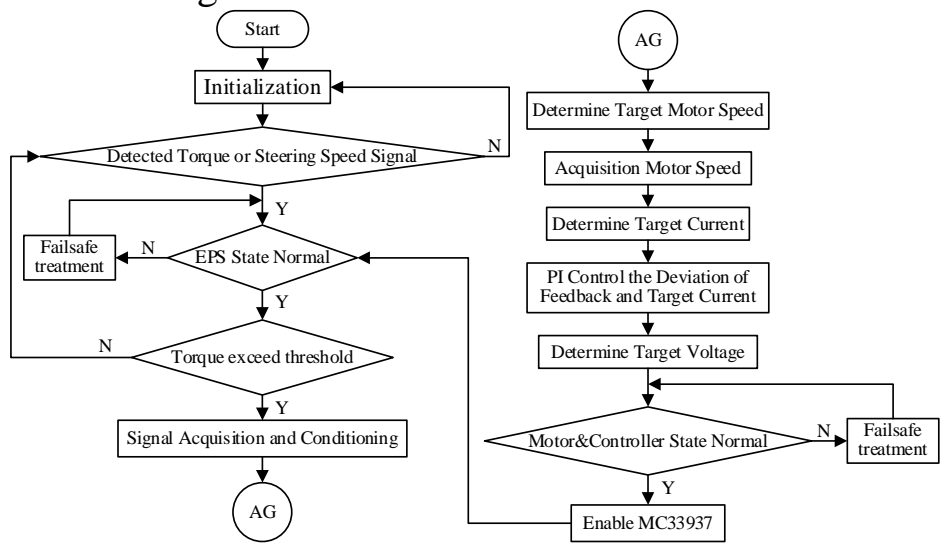

Fig. 6 Program flow chart

\section{Conclusions}

In this paper a new EPS controller has been designed based on MPC5602P according to the theory of induction motor vector control model. The debugging experiment indicated that it can satisfy the control function of EPS. And the motor speed control is highly responsive to the torque variation. Meanwhile, over-current, low-voltage and other failsafe can be solved in time. Ensure the safety of motor and controller. Thereby indicating the MPC5602P-based EPS design have significance to the high-performance controller development.

\section{Acknowledgments}

The authors would like to thank Shandong Province Science and Technology Development Project 2014GGX105006 for financial support.

\section{References}

[1] Minhui Zhu. The Future Technology of Electric Power Steering System. Automobile and Fittings, 2009, p. 17-19.

[2] Urich Sendler. INDUSTRIE 4.0. China Machine Press, 2014, p.28-50.

[3] Wenjiang Wu, Jinkui Hao. Electric Power Steering System Control System Design Based on MC9S08JM60. Micro Special Motors. Vol. 40 (2012) No. 7, p. 57-61.

[4] Pathak A D. MOSFET/IGBT DRIVERS THEORY AND APPLICATIONS. Application Note available on www. ixys. Com, 2001.

[5] Information on: http://www.freescale.com//. 